\title{
Digital Mammography - a new trend in breast carcinoma diagnostics
}

\author{
Slezak $\mathrm{P}^{1}$, Waczulikova $\mathrm{I}^{2}$ \\ Institute of Normal and Pathological Physiology, Bratislava, Slovakia. peter.slezak5@gmail.com
}

Comment on: Vanovcanova L, Lehotska V, Rauova K. Digital Mammography - a new trend in breast carcinoma diagnostics. Bratisl Lek Listy 2010; 111(9): 510-513.

L. Vanovcanova et al in their paper "Digital Mammography - a new trend in breast carcinoma diagnostics" published in Bratisl Lek Listy 2010; 111 (9): 510-513, have tried to determine the advantages of "digital mammography (over conventional mammography) in early detection of breast carcinoma". The importance of breast imaging techniques in accurate and early diagnosis of the disease is out of question. The study as a whole is comprehensible, well arranged, and strong on the practical side. As a result, it is certain that not just radiologists will find it useful. Nevertheless, the use of statistics in this work is questionable. The statistical analysis is flawed by actually being unfinished (there could have been done definitely more). Fortunately, the results of adequate statistical analysis even more support the conclusive statement made by the Authors (see below). Nevertheless, this cannot be used as an excuse for poorly performed statistical analysis because improper study design and analysis can be misleading.

The Authors state that collected data were statistically evaluated by the Mantel-Haenszel test. Please note that the MantelHaenszel method provides a pooled odds ratio across the strata (a series of fourfold tables). Apart from that, no results of this test are presented in the paper while in this study, the odds ratios are not what we are interested in. Moreover, no stratification is mentioned or presented in the study design.

To get a proper statistical analysis the Authors should have compared two independent proportions (1), namely that for digital mammography versus that for conventional mammography. We have performed a statistical re-evaluation of the data, and present the obtained results in Table 1 as follows.

Based on the results of our analyses we suppose that the Authors probably compared two independent proportions using con-

${ }^{1}$ Institute of Normal and Pathological Physiology, Bratislava, Slovakia, and ${ }^{2}$ Faculty of Mathematics, Physics and Informatics, Comenius University, Bratislava, Slovakia

Address for correspondence: P. Slezak, Institute of Normal and Pathological Physiology, Slovak Academy of Sciences, Sienkiewiczova 1, SK81371 Bratislava, Slovakia.

Phone: +421.2 .52926271 , Fax: +421.2 .52968516$ ventional normal approximation and not the Mantel-Haenszel test as stated in their Material and Methods. It can be anticipated that in order to compare results on the proportions between the groups (or modalities), the Authors used the number of detected cases as a total for each single group. Further they compared the proportions of detected Tis and T1 cases within the subgroups of diseased patients. In our opinion, this approach has actually masked the very intention of the Authors, namely to prove that the digital mammography "is capable to detect carcinomas in their early stages". Providing that i) the randomization had been properly done (see below), ii) the recall-rate proportion comprised "uncertain cases" who were not considered in "malignant-tumor rate", and iii) the null hypothesis is true (non-superiority of any modality), the proportions for the Negative, Uncertain, Tis, T1 and Other-stages categories should have had a similar pattern across the modalities for the original groups of patients.

Our further objections regard the study design. The Authors claim that a total of 11,799 patients were randomly assigned to the modalities; however, there is no mention as to which method of randomization they used. Despite the Authors' statement that "the selection was randomized and evenly distributed" there are more questions to be raised. Firstly, because the numbers of patients in the groups were not equal, and secondly, because the patient characteristics in both groups are missing, despite the fact that confounders are known at least in age and socioeconomic status, since they are associated with the endpoint (detection of breast carcinoma). Without this information, the test results cannot be unambiguously attributed to any of the investigated categorical characteristics (recall-rate, malignant-tumor rate, Tis and T1).

To conclude the appraisal part of our contribution, we would like to mention that the Authors could confidently have used onesided hypothesis for testing since they had a strong and supported reason to assume superiority of digital mammography over conventional one. This would have increased the power for detecting the effect(s). To summarize the re-analysis we can conclude that the difference between these two proportions is statistically significantly different from zero for two investigated variables, namely 
$123-124$

Tab. 1. The number of patients per modalities, and results of the statistical re-evaluation. Tis - stage of carcinoma in situ. The approximate confidence interval for the proportion difference was calculated by the Miettinen and Nurminen method (2).

\begin{tabular}{|c|c|c|c|c|c|}
\hline Modality & $\begin{array}{l}\text { Conventional } \\
\text { mammography }\end{array}$ & $\begin{array}{c}\text { Digital } \\
\text { mammography }\end{array}$ & $\begin{array}{c}\text { Proportion } \\
\text { difference }(\%)\end{array}$ & $\begin{array}{c}95 \% \text { confidence } \\
\text { interval }\end{array}$ & $\begin{array}{c}\text { Exact two } \\
\text { sided (mid) P }\end{array}$ \\
\hline Number of patients & 5664 & 6135 & & & \\
\hline Recall-rate & $538(9.50 \%)$ & $427(6.96 \%)$ & 2.54 & (1.54 to 3.54$)$ & $<0.0001$ \\
\hline Malignant tumors & $68(1.20 \%)$ & $98(1.60 \%)$ & -0.40 & $(-0.83$ to 0.03$)$ & $=0.0612$ \\
\hline Tis & $9(0.16 \%)$ & $17(0.28 \%)$ & -0.12 & $(-0.30$ to 0.06$)$ & $=0.1761$ \\
\hline T1 stage & $26(0.46 \%)$ & $45(0.73 \%)$ & -0.27 & $(-0.56$ to 0.01$)$ & $=0.0446$ \\
\hline
\end{tabular}

"Recall rate" and "T1 stage". Thus, a properly done analysis gives even more convincing evidence in favour of digital mammography than presented in the paper.

Nowadays, the necessity of appropriate use of statistical analysis as well as the importance of understanding the outcome of statistical procedures has become evident since an improper use of statistics is a big problem we face in medical research. Mishandled statistics threaten the validity and reliability of published studies but what is even more disturbing, such studies may unwittingly be used for further dissemination of biased and incorrect information within the medical field. Clinicians and other readers of medical journals should be able to critically appraise the methods used in the study because the reports on medical research are subjected also to the scrutiny of the whole readership. Therefore, it is indispensable to understand the value of statistics applied to medical research, as well as to be able to evaluate and appraise the context, validity and reliability of papers, reports and guidelines.

\section{References}

1. Fleiss JL, Levin B, Paik MCh. Statistical Methods for Rates and Proportions, 3rd Ed., J. Wiley 2003, 760 p.

2. Miettinen OS, Nurminen M. Comparative analysis of two rates. Statistics in Medicine 1985; 4: 213-226.

Received November 7, 2010. Accepted December 18, 2011. 